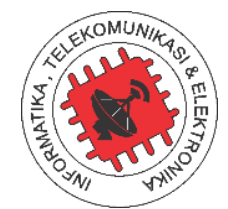

\title{
Analysis of User Throughput Based Predictive Mobility Load Balancing Using Logarithmic Regression of Reference Signal Received Quality In Long Term Evolution Radio Access Network
}

\author{
Achmad Rizal Danisya ${ }^{1}$, Rendy Munadi ${ }^{2}$, Sofia Naning Hertiana ${ }^{3}$ \\ ${ }^{1}$ Institut Teknologi Telkom Purwokerto \\ ${ }^{2,3}$ Universitas Telkom \\ ${ }^{1}$ Jl. D.I Panjaitan No.128 Purwokerto, Indonesia \\ 2,3J1. Telekomunikasi No. 1 Terusan Buah Batu, Bandung 40257, Indonesia \\ Corresponding email : achmad_rizal@ittelkom-pwt.ac.id
}

Received 27 December 2017, Revised 10 January 2018, Accepted 11 January 2018

\begin{abstract}
The improvement of Long Term Evolution (LTE) radio access network services is affecting the increased value of traffic load in its network, which is causing traffic unbalance between cells in LTE Radio Access Network (RAN). Users will be served with ineffective resource block allocation which will make the total of gained throughput are not optimal. A method is required to move network load from overloaded cells to underloaded cells in order to balance the resource block allocation optimally. By using NS-3.26 simulation, User Throughput Based (UTB) predictive Mobility Load Balancing (MLB) method is tested with RandomWalkMobilityModel for each user. This method produces an improvement of 2,29\% in average of total throughput of 63,33\% successful optimization.
\end{abstract}

Keywords - mobility load balancing, long-term evolution, logarithmic regression

Copyright $@ 2018$ JURNAL INFOTEL

All rights reserved.

\section{INTRODUCTION}

Embedded automation-intelligence in network equipment is required to manage more versatile subscriber's traffic nowadays, an immediate response of network parameters which is triggered by traffic behavior is useful for instant optimization of Quality of Service (QoS) and Quality of Experience (QoE). Preserving QoS and QoE are as important as maintaining the subscribers to stay in an operator's subscription. In 2011 SOCRATES project submitted an automated network management standardization which later is named Self Organizing Network (SON) standard [1]. That standard manages on how LTE equipment could optimize and improve its own network performance without or with minimal human mediation.

MLB is one of SON's feature which its duty is to balance network load between cells by steering User
Equipment(s) (UE) from overloaded cells to underloaded cells, this feature works by tweaking the RAN handover parameter(s) [2].

One of MLB benefit is to support the network stability even in the scenario of DDoS attack [3]. The MLB algorithm presents a mechanism which identifies eNodeB's cell state of congestion due to a DDoS attack or overloaded users, this ignites a procedure that can lead to the handover of some of its users to neighboring cells with sufficient resources.

From MLB algorithm research in [4], a significant gain in call blocking and cell-edge user throughput are produced with simple MLB mechanism which is based only on automatic adjustment of handover thresholds. From that research also shown that PRB utilization is not a sufficient indicator of cell load for MLB, even in the case of constant bit rate traffic. 
The method in [5] combines apriori expert knowledge with Multi-Objective Particle Swarm Optimization (MO-PSO), which allows to considerably reduce the search space and the computational time required for executing the MLB algorithm, this paper use offset parameters as its primary MLB consideration variable.

In [6] a practical multi-objective load balancing algorithm using Lagrange multiplier method was given. The simulation shows a significant improvement of Guaranteed Bit Rate of overall user satisfaction level and Best Effort users throughput in LTE networks by using the proposed algorithm.

In [7] the implementation of two measurement criteria is proposed as an improvement for the cell selection and handover procedures, cell's capacity estimation, and user speed. From the results shown, the proposed algorithm has the benefit of contributing to the macrocell offloading, network load balancing, and user QoS.

The objective of this paper is to give literature contribution in LTE predictive MLB method based on previous research [3-7]. RSRQ is one of LTE measurement parameters that will be analyzed as the primary parameter because its formula ingredients consist of SINR and network load value $(\rho)$, this presented in equation (7). The option to use the RSRQ is to simplify the time required for executing the MLB [5]. Mobility randomness from each UE is analyzed and predicted using logarithmic regression in every 5 seconds period of the simulation which has been done with different method in [6], this procedure is executed to anticipate the handover decision delay before UEs enter the handover threshold region. In [8] UE's handover decision to neighbor cell $j$ occurs when in some $t$ period the handover requirements are fulfilled, while this paper's proposed method is to predict the handover requirement in every certain period in order to maintain the network load balance in a respectable range and increasing the total throughput.

\section{RESEARCH METHOD}

\section{A. Simulation Design}

As can be seen in Fig. 1 the test scenario passed down in this paper is by providing 30 UEs in the overlapped area of 3 cells, which are cell 3,4 and 8 . This condition is designed in order to make each UE have 2 NeighbourCells's or 2 cells option for MLB handover. Every cell is assigned in the same frequency band or Frequency Reuse 1 (FR-1) [9] in $2100 \mathrm{MHz}$ with 25 Resource Block or $5 \mathrm{MHz}$ bandwidth configuration. Used Antenna system is MIMO $2 \times 2$ $\left(N_{T}=2 ; N_{R}=2\right)$.

All of this setting are then evaluated using NS-3.26 in 30 iterations, wherein each iteration the location of each UEs are pseudo-randomized between $0-500 \mathrm{~m}$ range and the UE speed also pseudo-randomized in the range between $1-23 \mathrm{~m} / \mathrm{s}$. All of the pseudorandomization processes are uniformly distributed.

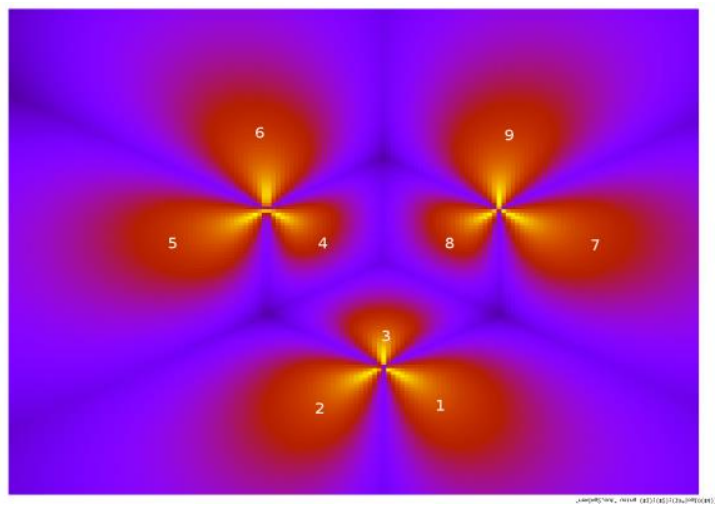

Fig.1. Radio Environment Map in Simulation Topology

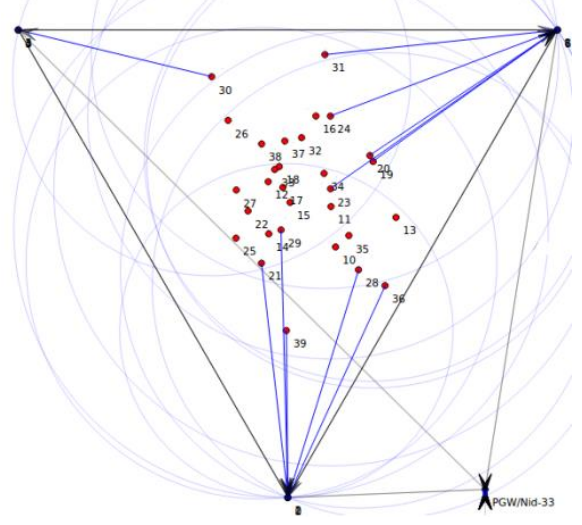

Fig.2. UE Allocation in Simulation Topology

The handover algorithm run in this paper is the A2A4RsrqHandoverAlgorithm [10]. RSRQ is chosen because it contains two network QoS parameters which are SINR and traffic load $\rho_{i}$. The relationship between RSRQ, SINR and $\rho_{i}$ presented in the following equation [11].

$$
R S R Q=\frac{\operatorname{SINR}}{12\left(1+\operatorname{SIN} R \times \rho_{i}\right)}
$$

SINR value is defined as [12]

$$
S I N R=\frac{S_{i}}{N+\sum_{j} I_{j}}
$$

and cell $i$ network load, $\rho_{i}$, is defined as [8].

$$
\rho_{i}=\sum_{k} \frac{n_{k}}{K \Delta T}
$$

$S_{i}$ is the measured received signal power by a UE from a ServingCell $i, N$ is the noise power suffered and $I_{j}$ is the interference from cell $j$ which is sensed by the UE in ServingCell $i . n_{k}$ is the Physical Resource Block (PRB) number which is allocated for bearer $k$ from possible of $K \mathrm{PRB}$ in a measurement period of $\Delta T$ subframe. Conducted experiment was using duplex traffic of User Datagram Protocol (UDP) packets with constant sized packets ( $L$ ) set 
to 8 Kilobit/packet. The transmit interval is $1 \mathrm{~ms}$ which produce an arrival rate $(\lambda)$ of 1000 packet/s. so the network demand from each UE is $\rho_{u}=\lambda L=$ $8 \mathrm{Mbps}$. For the specification of each UE's Evolved Packet System (EPS) bearer is Guaranteed Bit Rate (GBR) with QCI-3 which has delay budget of $50 \mathrm{~ms}$ [13].

\section{B. Simulation Algorithm}

This paper applies an intra-frequency handover in the LTE RAN which occurs when the following conditions are met [4].

$$
M_{j}-M_{i}>O_{i}+\xi+\eta
$$

With subscript $j$ shows the affiliation with NeighbourCell which will be the handover target and subscript $i$ is the affiliation of ServingCell, $M$ is the measurement parameter of radio signal quality (RSRQ or SINR) or strength (RSRP) which is sensed by the UE. $O_{i}$ is the primary offset belongs to cell $i$, which will make the measurement value of ServingCell $O_{i} \mathrm{~dB}$ larger than the real value. The purpose of this parameter is to ensure that the NeighbourCell $j$, which is the handover target, has higher value to avoid the ping-pong effect after the handover occurs [2]. $\xi$ is the hysteresis value and $\eta$ is the global offset which is determined for the whole network, both parameters are ignored for this paper analysis because they are not configurable in the simulation. So (4) can be simplified to (5).

$$
M_{j}>M_{i}+O_{i}
$$

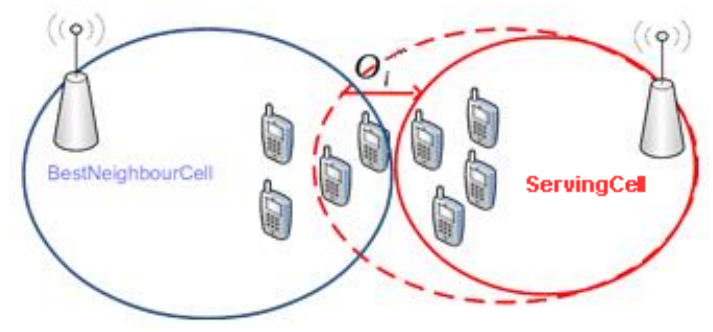

Fig.2. Effect CellIndividualOffset Variation

The determination of $O_{i}$ value so that (some) $\mathrm{UE}(\mathrm{s})$ can be offloaded from cell $i$ to cell $j$ is the focal point in this paper. On fig. 2 it can be seen that by changing the amount of $O_{i}$ the cell coverage can be expanded or narrowed. If the value of $O_{i}$ is decreased then the coverage will be decreased and vice versa if $O_{i}$ is increased. The decision to determine $O_{i}$ is obeying the consideration of RSRQ value from the target cells. Steps that has been done to evaluate the predictive MLB in each iteration are,

a) Get each cell's total throughput results then determine which cell is the most overloaded and underloaded. A cell is considered overloaded if it has a total throughput that conforms to this condition.

$$
T h r_{i}>T h r_{a v} \times 1,1
$$

Where $T h r_{a v}$ is the average throughput of all cells which are involved in MLB process, while the following equation is the definition of throughput.

$$
T h r_{i}=\frac{R x_{i}}{T}
$$

With $R x_{i}$ is the number of received bits from cell $i$ in a period of $T$.

b) For initial 5 seconds period do the RSRQ time series logarithmic regression for every UE's towards to all cells that cover it. Variable $a$ and $b$ for curve fitting are obtained from that initial statistic data [14].

$$
\aleph_{u, i}=f_{u, i}(t)=a+b \ln (t)
$$

Equation (8) is exploited to predict the RSRQ of UE $u$ on cell $i$ for the next 5 second period. Equation (9) until (13) are handy to find the component of $\aleph_{u, i}$ [14].

$$
\begin{aligned}
& S_{X X}=\sum_{i}\left(x_{i}-\bar{x}\right)^{2} \\
& S_{Y Y}=\sum_{i}\left(y_{i}-\bar{x}\right)^{2} \\
& S_{X Y}=\sum_{i}\left(x_{i}-\bar{x}\right)\left(y_{i}-\bar{y}\right) \\
& b=S_{X Y} / S_{X X} \\
& a=\bar{y}-b . \bar{x}
\end{aligned}
$$

c) After the curve fitting components of $\aleph_{u, i}$ are obtained then next step is to get RSRQ expectation value $\left(E\left[\aleph_{u, i}\right]\right)$ for the next 5 seconds period. So for $t_{1}=t_{0}+5 \mathrm{~s}$ the expectation value of RSRQ in period of $t_{0}$ until $t_{1}$ can be predicted from.

$$
\begin{aligned}
& E\left[\aleph_{u, i}\right]=\frac{1}{T_{s}} \int_{t_{0}}^{t_{1}}(a+b \ln (t)) d t= \\
& \frac{1}{T_{s}}\left[a\left(t_{1}-t_{0}\right)+b\left(t_{1} \ln \left(t_{1}\right)-t_{0} \ln \left(t_{0}\right)-t_{1}+\right.\right. \\
& \left.\left.t_{0}\right)\right]
\end{aligned}
$$

d) After step 3 the RSRQ value from every UE towards cells that govern are grouped based on its ServingCell, then the value of $\beth_{u-i, j}$ is obtained from group of UEs which it's ServingCell gets overload criteria.

$$
E\left[\aleph_{u, j}\right]-E\left[\aleph_{u, i}\right]=\beth_{u, j}-\beth_{u, i}=\beth_{u-i, j}
$$

Where,

$$
\begin{aligned}
\beth_{u-i, j}= & \text { Difference of RSRQ expectation between } \\
& \text { UE } u \text { on underloaded NeighbourCell } j \\
& \text { with UE } u \text { in overloaded ServingCell } i \\
& \text { in period of } t_{0} \text { until } t_{1} . \\
\beth_{u, i}= & \text { RSRQ expectation of UE } u \text { in overloaded } \\
& \text { ServingCell } i \text { in period of } t_{0} \text { until } t_{1} . \\
\beth_{u, j}= & \text { RSRQ expectation of UE } u \text { in } \\
& \text { underloaded NeighbourCell } j \text { in period } \\
& \text { of } t_{0} \text { until } t_{1} .
\end{aligned}
$$

e) Then the values of $\beth_{u-i, j}$ are sorted descending, if there are no positive value then $O_{i}$ 
is set to 0 according to Table 9.1 .7 in [15]. If there are $\beth_{u-i, j}$ with positive value then the determination of the value of $O_{i}$ depends on the estimation of UE $u$ transferred load from cell $i$ to cell $\mathrm{j}\left(\rho_{u, j}\right)$, which can be calculated from [11] in (16)

$$
\rho_{u, j}=\frac{1}{12 \times \aleph_{u, j}}-\frac{1}{\operatorname{SIN} R_{u, i}}
$$

but with a limitation of

$$
1>\rho_{j}=\rho_{j}+\sum_{u} \rho_{u, j}
$$

with $\rho_{j}$ itself is the network load of NeighbourCell $j$ and $S I N R_{u, i}$ is SINR value from UE $u$ towards ServingCell $i$.

\section{Analysis Parameters}

After all of 30 iterations conducted there are 3 parameters that will be analyzed to assess the performance of the MLB method, those are.

a) Throughput Gain $\left(G_{T h r}\right):$ The cell's throughput difference between simulation result with MLB $\left(T h r_{M L B}\right)$ and simulation result without MLB (Thr $\left.r_{\text {Normal }}\right)$ compared by cell's throughput of simulation result without MLB.

$$
G_{T h r}=\left(\frac{T h r_{M L B}-T h r_{\text {Normal }}}{T h r_{\text {Normal }}}\right)
$$

b) Throughput deviation $\left(\sigma_{T h r}\right)$ : The difference between maximum and minimum throughput from all of the cells involved in MLB process divided by average throughput $(\overline{T h r})$ from all of the cells involved in MLB process :

$$
\sigma_{T h r}=\frac{\max (T h r)-\min (T h r))}{\overline{T h r}}
$$

c) Deviation Gain $\left(G_{\sigma}\right)$ : difference between throughput deviation without MLB $\left(\sigma_{T h r-N o r m a l}\right)$ and throughput deviation with MLB $\left(\sigma_{T h r-M L B}\right)$.

$$
G_{\sigma}=\sigma_{T h r-N o r m a l}-\sigma_{T h r-M L B}
$$

Parameter a) is used to see how well the MLB algorithm improving the network bandwidth utilization and the next 2 parameters are used to see how well the predictive MLB algorithm distributing the network load amongst the cells involved.

\section{RESULT}

Figure 3 shows the simulation result from each of iteration, and Fig. 4 showed that some iteration produced positive and negative $G_{T h r}$. From 30 iterated simulation the average of throughput gain $\left(\overline{G_{T h r}}\right)$ is $1,21 \%$ with optimization success rate of $63,33 \%$ or 19 iteration scenario has been optimized successfully.

The definition of throughput optimization success rate is the comparison between the number of iteration that produce $G_{T h r}>0$ with the total number of iteration. If the iteration data are only averaged for
$G_{T h r}>$ the value of $\overline{G_{T h r}}$ increase to $2,21 \%$, it means that if this method is applicable than the expectation is $2,21 \%$ of optimization. The result is close to [4], [10] which has not predictive yet.

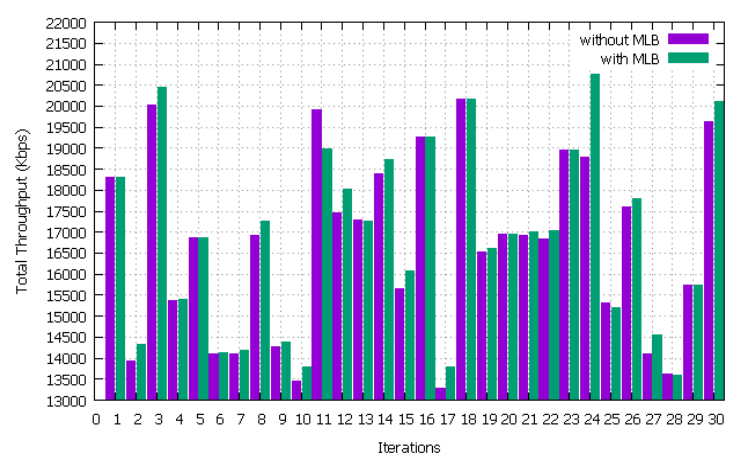

Fig.3. Total Throughput For 30 Iterations of Simulation

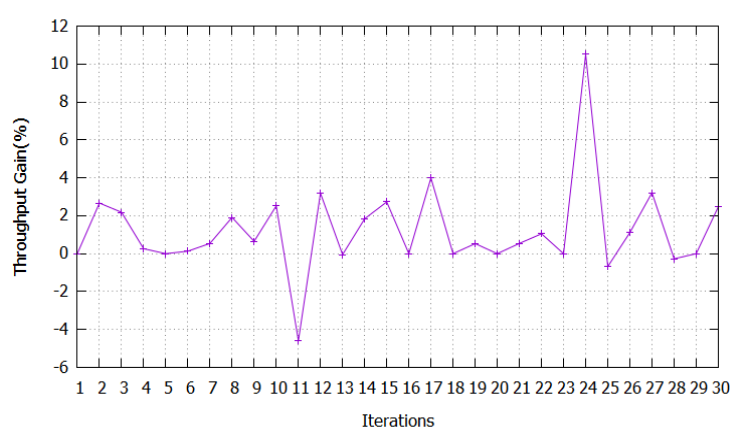

Fig.4. Throughput Gain in \% For 30 Iterations of Simulation

From Fig.5 positive valued $G_{\sigma}$ means that MLB throughput deviation result got smaller compared to before predictive MLB applied which also means that network load balancing is achieved, but from the iterations statistic, only $40 \%$ of iteration produced positive $G_{\sigma}$. The average of deviation gain for all of the iteration is $-0,26 \%$ which means that the network load unbalance increase using this load balancing method.

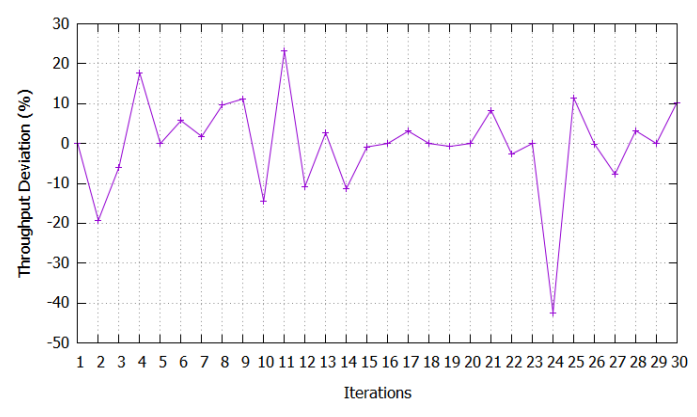

Fig.5. Throughput Deviation in \% For 30 Iterations of Simulation

\section{DISCUSSION}

There are 11 from 30 iterated simulation scenario or $36,67 \%$ of overall iteration are not optimizable which could be caused by some factors, which are,

a) Randomized UE's velocity, direction, and position for each of the scenario allow the UE to 
be in a position which causes the predicted RSRQ difference between NeighbourCell and ServingCell are not fulfilling (5). This affected the calculation of the simulation for attenuation and interference between UE $u$ with every cells that govern. the calculation is for SINR determination [13] and in accordance to (16) SINR value will affecting RSRQ value directly.

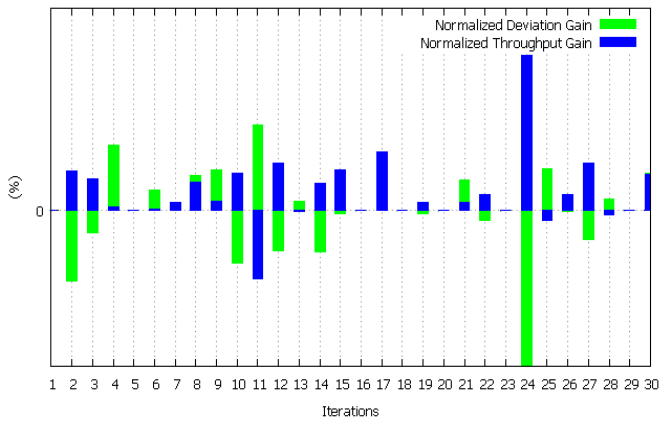

Fig.6. Normalized Deviation Gain vs Normalized Throughput Gain

b) Referencing radio network throughput as the radio resource availability parameter is not an adequate way to assume a cell/node needs to be load balanced. The implication can be shown from fig. 6 which shows that $73.33 \% G_{T h r}$ are inversely proportional to $G_{\sigma}$. The most extreme point is at 24th iteration, where maximum normalized $G_{T h r}$ (to maximum $G_{T h r}$ - which is $10,51 \%$ ) is directly statistic related to the most minimum normalized $\sigma_{T h r}$ (to maximum of absoluted $\sigma_{T h r}$ - which is $42.4 \%$.).

c) An example of how the MLB algorithm works are presented in Fig.8 and Fig.9. The figure comes from 17 th iteration where the simulation produces positive throughput gain and deviation gain. In Fig.7 at $t=5 \mathrm{~s}$ because the default $O_{i}$ is set to $2,5 \mathrm{~dB}$ then UE 24 is still attached to cell 3 when it should have moved to cell 4 . This decision made UE 24 fail to get an optimal Modulation Coding Scheme (MCS) with the granted SINR from cell 3. The 5 steps MLB algorithm then calculated for 17 th iteration, from the logarithmic regression the value of the new $O_{i}$ is decided to be $0 \mathrm{~dB}$ because there are no positive value from the list of $\beth_{u-i, j}$, This can be seen from Fig.7.

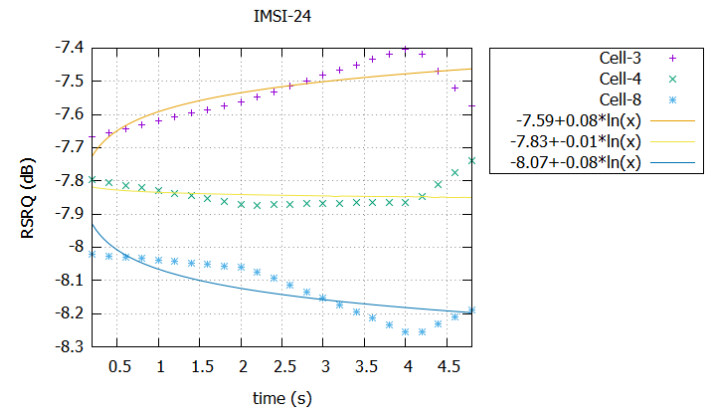

Fig.7. Logarithmic Regression of UE with IMSI-24's RSRQ
Then when MLB applied in the same period of time for UE 24 just like what Fig.9 have shown, It will gain more optimal RSRQ value which is related to better SINR and then better MCS which resulting in a higher throughput gain [13].

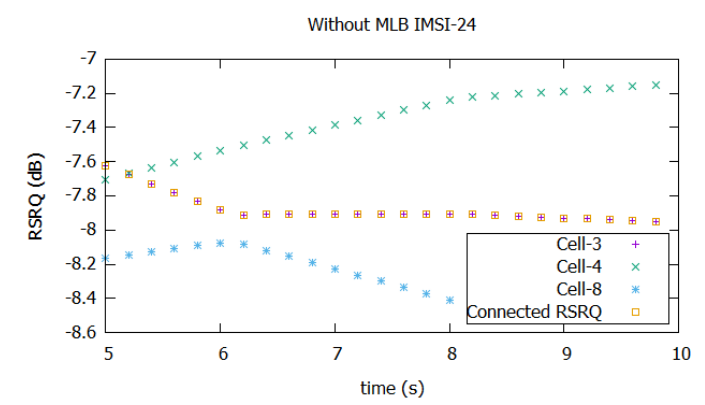

Fig.8. RSRQ of UE with IMSI-24 without MLB

But with MLB process that reduces NeighbourCellOffset $O_{i}$ to make as if a cell is lowering it's coverage. It will give a higher probability for UE, which already have a critical

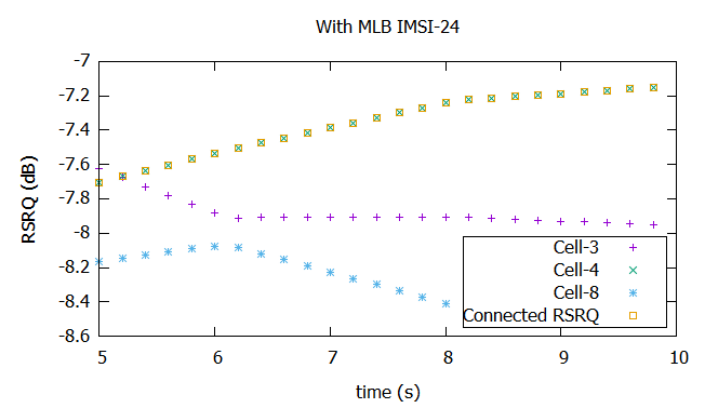

Fig.9. RSRQ of UE with IMSI-24 with MLB

SINR in ServingCell's, cell to be moved unto a NeighbourCell with a more risky cell offset guarantee. This will cause the target cell (in this case a certain NeighbourCell $j$ ) who receives UE from ServingCell $i$ in order to raise its total cell throughput will decrease instead. The cause is the addition of the UE with a worse SINR results a decrease in averaged MCS in the new ServingCell. Decreased modulation order results in decreased throughput compared to the initial Serving Cell.

\section{CONCLUSION}

The purpose of this research is to simulate a predictive load balancing method in LTE network based on imbalance throughput between cells while still considers the signal quality and cell capacity of the destination cells with only one parameter observation. From the evaluation, the optimum value in terms of $G_{\sigma}$ has not been obtained although the averaged throughput gain ( $\overline{G_{T h r}}$ ) for all of the scenarios is $2,21 \%$, which is not surpassing the best results from previous researches. Offset selection process requires a more advanced calculation to determine the composite value of $\rho_{j}$. Signal quality 
(SINR) predictions also requires longer periods of data for time series analysis. Longer period of statistical sample implies a need for more complex (yet valid) and accurate time series signal modeling to compensate more random signal characteristics. A good load balancing method is a method that able to increase the total throughput, decrease, or at least maintain, the throughput deviation and has high success rate of optimization for cells which are involved in load balancing process.

\section{REFERENCES}

[1] L. Christoph and S. Nokia, "Eu Fp7 Strep Socrates," pp. 700-700.

[2] S. Hämäläinen, H. Sanneck, and C. Sartori, LTE SelfOrganising Networks (SON): Network Management Automation for Operational Efficiency. 2012.

[3] S. Zinno, G. Di Stasi, S. Avallone, G. Ventre, and N. Federico, "A Load Balancing Algorithm against DDoS Attacks in Beyond 3G Wireless Networks," 2009.

[4] R. Kwan, R. Arnott, R. Paterson, R. Trivisonno, and M. Kubota, "On mobility load balancing for LTE systems," IEEE Veh. Technol. Conf., no. 3, pp. 2-6, 2010.

[5] Z. Altman, S. Sallem, R. Nasri, B. Sayrac, and M. Clerc, "Particle swarm optimization for Mobility Load Balancing SON in LTE networks," 2014 IEEE Wirel. Commun. Netw. Conf. Work. WCNCW 2014, no. 1, pp. 172-177, 2014.

[6] C. Gang, M. Fanfan, and S. Li, "QoS-priority based load balancing algorithm for LTE systems with mixed users," J. China Univ. Posts Telecommun., vol. 22, no. 3, pp. 9-17, 2015.

[7] E. J. C. De La-roque, C. Patrick, C. Renato, and L. Francês, "A New Cell Selection and Handover Approach in Heterogeneous LTE Networks Additional Criteria Based on Capacity Estimation and User Speed," no. c, pp. 57-65, 2015.

[8] S. Hahn, D. M. Rose, and T. Kürner, "Mobility Load Balancing - A Case Study: Simplified vs . Realistic Scenarios," Euro-Cost, 2014.

[9] A. Hikmaturokhman, V. Lutfita, and A. R. Danisya, “4G-LTE $1800 \mathrm{Mhz}$ coverage and capacity network planning using Frequency Reuse 1 model for rural area in Indonesia," in ACM International Conference Proceeding Series, 2017.

[10] ns-3 Model Library. 2016.

[11] X. Zhang, LTE Optimization Engineering Handbook. 2017.

[12] T. Yamamoto, T. Komine, and S. Konishi, "Mobility Load Balancing Scheme based on Cell Reselection," Eighth Int. Conf. Wirel. Mob. Commun. Mobil., no. c, pp. 381-387, 2012.

[13] S. Dahlman, Parkvall, 4G LTE-Advanced for Mobile Broadband. 2011.

[14] C. R. Shalizi, "Advanced data analysis from an elementary point of view," B. Manuscr., p. 801, 2013.

[15] T. Specification, "TS 136133 - V12.7.0 - LTE; Evolved Universal Terrestrial Radio Access (EUTRA); Requirements for support of radio resource management (3GPP TS 36.133 version 12.7.0 Release 12), "vol. $0,2015$. 